Received: 24 October 2016

Accepted: 12 July 2017

Published online: 25 August 2017

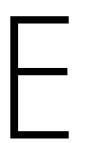

SCE

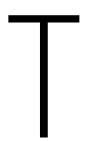

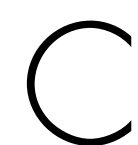

REP

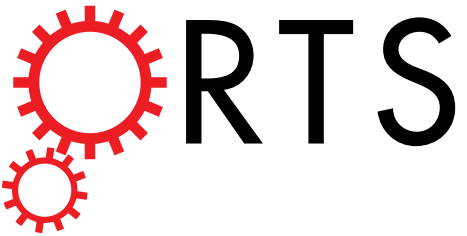

OPEN

Changing Epidemic of HIV and Syphilis Among Resident and Migrant Men Who Have Sex with Men in Jiangsu, China

Yuheng Chen ${ }^{1}$, Weiming Tang ${ }^{2}$, Lusi Chen ${ }^{3}$, Lingen Shi ${ }^{1}$, Xiaoyan Liu ${ }^{1}$, Jinshui X ${ }^{1}{ }^{1}$, Haiyang $\mathrm{Hu}^{1}$, Haitao Yang ${ }^{4}$, Xiping Huan ${ }^{1}$ \& Gengfeng $\mathrm{Fu}^{1}$

Men who have sex with men (MSM) in China face high rates of HIV and syphilis infection exacerbated by internal migration. Studies on the differences of HIV and syphilis epidemics changing trends in high-risk behaviors and geographic distribution between resident and migrant MSM in Jiangsu, China were conducted. MSM were recruited from 14 surveillance sites in the serial cross-sectional study. Data on demographics, sexual behaviors, HIV and syphilis prevalence were collected. Participants were classified as residents or migrants based on household registration. During 2010-2014, 19,750 MSM were investigated. Engaged in anal sex $(76.3 \%$ to $80.2 \%, \mathrm{P}<0.01)$ as well as received HIV-related services $(72.1 \%$ to $79.2 \%, \mathrm{P}<0.01)$ were increasing. In contrast, engaged in commercial anal sex with males $(7.4 \%$ to $5.0 \%, \mathrm{P}<0.01)$ and drug use $(1.6 \%$ to $0.8 \%, \mathrm{P}<0.01)$ were decreasing. HIV prevalence ranged between $8.6 \%$ to $9.6 \%$, while syphilis prevalence decreased over time $(13.4-6.8 \%, P<0.01)$. Further, we found that migrant MSM were more likely to engage in condomless anal sex, also had a higher HIV and syphilis prevalence than resident. During the study period, while syphilis prevalence decreased, higher rates of risk behaviors among migrant MSM called for targeted intervention strategies to reduce the HIV transmission.

Men who have sex with men (MSM) represent a high-risk group for the transmission of HIV/AIDS and syphilis, mainly due to low rates of condom use and high rates of multiple partners ${ }^{1}$. Over the past few years, MSM have become the highest-risk group for HIV infection in $\mathrm{China}^{2}$, with the rate of HIV infection rising annually. For example, the HIV/AIDS prevalence among MSM increased from 2.5\% in 2006 to $7.8 \%$ in $2014^{3}$. The prevalence of

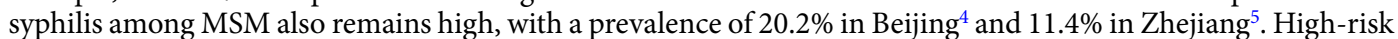
behaviors and lower coverage of preventive measures may be the potential driving force in the spread of HIV and sexually transmitted infections (STI $)^{6-8}$.

It was estimated that there are more than 245 million internal migrants in China, accounting for $20 \%$ of the total population in $2013^{1}$. Migration in China seems to be temporary and unstable ${ }^{9}$. The majority of migrants do not have legal permanent urban residency status and receive less social welfare such as medical and educational benefits than permanent residents ${ }^{10}$. Previous studies have demonstrated that migration may be an important factor to the spread of HIV/STIs in China ${ }^{11-13}$. Migration functions as a "bridge" with lax social control, social isolation, and selective migration ${ }^{14}$, allowing the spread of HIV/STIs from high-risk groups to general population $^{15}$. Furthermore, an epidemiologic study indicated that migration is more common among MSM, while migrant MSM are more likely to provide sexual services for money and engage in condomless sex, as compared with resident $\mathrm{MSM}^{16,17}$. Because of the better employment opportunities, more comfortable living environment, and less discrimination ${ }^{18}$, large metropolitan and developed areas are more likely to attract MSM $^{19}$. As one of the most densely populated and economically developed areas in eastern China, Jiangsu borders other less developed

${ }^{1}$ Institute for STI and HIV Control and Prevention, Jiangsu Provincial Center for Disease Control and Prevention, Nanjing, Jiangsu, 210009, China. 'University of North Carolina Project-China, Guangzhou, 510095, Guangdong, China. ${ }^{3}$ School of Public Health Southeast University, Nanjing, Jiangsu, 210009, China. ${ }^{4}$ Jiangsu Institute of Parasitic Diseases, Wuxi, Jiangsu, 214064, China. Yuheng Chen and Weiming Tang contributed equally to this work. Correspondence and requests for materials should be addressed to X.H. (email: huanxp@vip.sina.com) or G.F. (email: fugf@jscdc.cn) 
provinces and attracts a large number of MSM migrants ${ }^{20}$. The high rates of migration and sexual risk behaviors among MSM are challenges for HIV/STIs control in China. More control efforts should focus on migrant MSM, who are vulnerable to HIV/STIs.

This study aimed to evaluate the changing trends of HIV and syphilis epidemics, and risk behaviors among resident and migrant MSM in Jiangsu, China.

\section{Study Methods}

Sample size. Based on WHO recommendations and experience from other countries ${ }^{21-23}$, as well as the suggestion of Chinese CDC, a minimum sample size of 250 to 400 participants was required for each essential surveillance site. The sample size calculation has been reported elsewhere ${ }^{18}$. In short, the sample size was calculated using the following formula: $\mathrm{N}=\left[4 * \mathrm{z}_{\alpha}{ }^{2} * \mathrm{P}(1-\mathrm{P})\right] / \mathrm{W}^{2}$, wherein, $\mathrm{z}_{\alpha}$ is a factor that corresponds to the desired confidence interval (for a $95 \%$ confidence level, $\mathrm{z}_{\alpha}=1.96$ ); $\mathrm{P}$ is the expected proportion of patients with the outcome; $\mathrm{W}$ is the width of the interval, for example, the width for a margin of error of $+/-3 \%$ is $0.06^{22,23}$.

Recruitment and Ethical Approval. Data collections were conducted between April and August of each year from 2010 through 2014. To be eligible, participants needed to be at least 18 years old, born biologically male and report having oral or anal sex with men in the previous year. MSM who had been diagnosed HIV positive in the past should also be incorporated into the survey. Convenience sampling methods (snowball sampling, venue-based sampling, and online recruitment) were used to recruit participants. Investigators within the MSM community found potential subjects in MSM activity venues, including commercial entertainment locations (bars and clubs) and public places (parks and public restroom) and invited them to participate in the study. Recruitment information was also placed on Internet forums, online chat rooms, and instant messaging software used by MSM. To ensure the quality of the survey, in each of the study site, the same sampling design and study protocol, which include comprehensive quality control plan, were followed and staff were trained as per the same training module.

The survey was approved by the ethics committees of the Jiangsu Provincial Center for Disease Control and Prevention (JSCDC). A signed informed consent was obtained from each participant for the questionnaire survey and blood collection. Each participant was reminded of the right to decline participation or quit the study at any time. All methods were performed in accordance with the relevant guidelines and regulations that were approved by the Ethical Committee of Jiangsu CDC.

Demographic and Behavioral Measures. We collected information regarding demographics, sexual risk behaviors, drug use (refers to traditional drugs) and the receiving HIV-related services from the participants by using a structured, self-administered questionnaire. According to the self-reported official household registration, participants were classified as residents or non-residents (migrants). If participants reported that they were from a different province or foreign country, they were defined as migrants. Otherwise, participants were considered as residents, consistent with similar past studies ${ }^{24-26}$. Demographic questions included age, marital status, education level, the length of stay in the local area, and common venues for finding partners.

Participants were asked to recall their sexual activities and behaviors in the last six months, including non-commercial sex with males, commercial anal sex with a male, vaginal sex with a female, and use of a condom during these sexual activities. Other behaviors, including injection and non-injection drug use, were also included in the questionnaire. Participants were also asked about their exposure to HIV/AIDS preventive services, including free condom services, HIV/AIDS counseling and detection, methadone maintenance therapy, clean needle exchange and peer education. Participants who had accepted one of these services in the last year were categorized as having received HIV/AIDS related services.

Biological testing for HIV and Syphilis. Five ml. of venous blood (anticoagulated) was collected from each participant for HIV and syphilis antibody testing, using the standard protocol and laboratory methods ${ }^{27}$. HIV antibodies were screened using a rapid test (Acon Biotec Co., Ltd), with positive screening tests confirmed using Western Blot (WB, HIVBLOT 2.2, Genelabs Diagnostics, Singapore). Syphilis antibodies were screened using the ELISA (Wantai Biopharmacy Co., Ltd) test and confirmed with TRUST (Wantai Biopharmacy Co., Ltd) ${ }^{28}$.

Statistical analysis. All data were entered into the HIV/AIDS Sentinel Surveillance Network server using client software. After data had been exported and cleaned, SAS version 9.2 ${ }^{29}$ and SPSS 23.0 were used for statistical analyses. Descriptive analysis was conducted to compare demographic characteristics, sexual behaviors, drug use, and use of HIV-related services, as well as to compare HIV and syphilis infection rates between resident and migrant MSM. The Cochran-Armitage trend test was used to examine the trend associated with these risk factors during the study period. Univariable and multivariable logistic regression models were conducted to compare the HIV/syphilis prevalence and related sexual risk behaviors, by comparing the data collected in 2014 to 2010. In univariate analysis, each infection (HIV/syphilis) and various risk behaviors were defined as dependent variables, while time was treated as an independent variable. Multivariate logistic regression adjusted for age, marital status and education. Crude odd ratios and adjusted odd ratios (AOR), as well as their $95 \%$ confidence intervals $(95 \% \mathrm{CI})$, were calculated to demonstrate the association between time and factors. Chi-square statistics were applied to test the differences between factors associated with sexual risk behaviors for HIV positive and negative individuals in each surveyed year. Epi Info software was used to determine and demonstrate the geographical distribution of the epidemics. Electronic maps were obtained from Jiangsu CDC. 


\begin{tabular}{|c|c|c|c|c|c|c|c|c|c|c|c|c|}
\hline \multirow[b]{3}{*}{ Characteristics } & \multicolumn{2}{|l|}{2010} & \multicolumn{2}{|l|}{2011} & \multicolumn{2}{|l|}{2012} & \multicolumn{2}{|l|}{2013} & \multicolumn{2}{|l|}{2014} & \multicolumn{2}{|l|}{ Total } \\
\hline & \multicolumn{2}{|l|}{$(\mathrm{N}=3061)$} & \multicolumn{2}{|l|}{$(\mathrm{N}=3337)$} & \multicolumn{2}{|l|}{$(\mathrm{N}=4380)$} & \multicolumn{2}{|l|}{$(\mathrm{N}=4208)$} & \multicolumn{2}{|l|}{$(\mathrm{N}=4766)$} & \multicolumn{2}{|l|}{$(\mathrm{N}=19750)$} \\
\hline & Resident \% & Migrants\% & Resident \% & Migrants\% & Resident \% & Migrants\% & Resident \% & Migrants \% & Resident \% & Migrants\% & Resident \% & Migrant $\%$ \\
\hline $\begin{array}{l}\text { Percentage of the } \\
\text { class }\end{array}$ & 75.6 & 24.4 & 79 & 21 & 82.7 & 17.3 & 80.7 & 19.3 & 80.5 & 19.5 & 80 & 20 \\
\hline \multicolumn{13}{|l|}{ Age (years) } \\
\hline$<20$ & 5 & 4.8 & 6.8 & 4.6 & 5.1 & 4.9 & 6.1 & 5.9 & 4.6 & 8.1 & 5.4 & 5.8 \\
\hline $20-29$ & 45.1 & 56.9 & 50.4 & 63.6 & 48.5 & 56 & 48.1 & 61.2 & 48.3 & 56.6 & 48.2 & 58.7 \\
\hline $30-39$ & 27.5 & 25.6 & 23.3 & 22.3 & 25.8 & 27.1 & 25.9 & 23.2 & 23.5 & 21 & 25.1 & 23.7 \\
\hline $40-49$ & 16.7 & 9.5 & 14 & 7.4 & 14.3 & 9.6 & 13.2 & 6.9 & 15.3 & 9.5 & 14.6 & 8.6 \\
\hline$\geq 50$ & 5.7 & 3.2 & 5.5 & 2.1 & 6.5 & 2.4 & 6.7 & 2.7 & 8.3 & 4.8 & 6.7 & 3.1 \\
\hline \multicolumn{13}{|l|}{ Marital status } \\
\hline Single & 58.6 & 64.8 & 63.3 & 71.4 & 56.8 & 66.6 & 60.6 & 73 & 56.1 & 72.3 & 58.8 & 69.7 \\
\hline Married & 33.9 & 28 & 32 & 26 & 38.8 & 31.2 & 33.2 & 22 & 38.3 & 22.7 & 35.6 & 25.8 \\
\hline Divorced/Widowed & 7.5 & 7.3 & 4.7 & 2.6 & 4.4 & 2.2 & 6.2 & 5.1 & 5.6 & 5.1 & 5.6 & 4.5 \\
\hline \multicolumn{13}{|c|}{ Highest level of education } \\
\hline $\begin{array}{l}\text { Junior high school } \\
\text { or lower }\end{array}$ & 22.8 & 34.5 & 19.4 & 22.6 & 21.9 & 28.2 & 21.5 & 30.5 & 18.1 & 26.8 & 20.6 & 28.5 \\
\hline $\begin{array}{l}\text { Senior high school } \\
\text { or above }\end{array}$ & 77.2 & 65.5 & 80.6 & 77.4 & 78.1 & 71.8 & 78.5 & 69.5 & 81.9 & 73.2 & 79.4 & 71.5 \\
\hline \multicolumn{13}{|c|}{ Length of time living in current location } \\
\hline Less than one year & 19.9 & 47.3 & 18.5 & 39.1 & 12.5 & 35.9 & 11.2 & 37.8 & 8.8 & 36 & 13.4 & 39 \\
\hline One year or more & 80.1 & 52.7 & 81.5 & 60.9 & 87.5 & 64.1 & 88.8 & 62.2 & 91.2 & 64 & 86.6 & 61 \\
\hline \multicolumn{13}{|c|}{ Partner seeking Venues } \\
\hline Internet & 24.5 & 21.4 & 34.5 & 36.7 & 47.6 & \begin{tabular}{|l|}
40.7 \\
\end{tabular} & 40.2 & 24.3 & 62.5 & 29.3 & 44 & 30.3 \\
\hline Bar/Club & 35.4 & 26.2 & 23.9 & 18.6 & 29.2 & 27.8 & 36.6 & 39.3 & 18.6 & 31.3 & 28.2 & 29 \\
\hline Bath center & 24.7 & 36.9 & 24 & 28.4 & 16.5 & 26.4 & 7.3 & 11.9 & 6.7 & 16.1 & 14.6 & 23.3 \\
\hline Public places & 0.4 & 0.1 & 2.2 & 0.6 & 0.6 & 0.5 & 2.8 & 2.7 & 0.2 & 0.2 & 1.2 & \begin{tabular}{|l|} 
\\
\end{tabular} \\
\hline Other & 15 & 15.3 & 15.3 & 15.7 & 6.1 & 4.6 & 13.1 & 21.8 & 11.9 & 23.2 & 11.9 & 16.5 \\
\hline
\end{tabular}

Table 1. Demographic Characteristics of MSM Recruited Between 2010 and 2014 in Jiangsu $(\mathrm{N}=19,750)$.

\section{Results}

Demographic Characteristics. From 2010 to 2014, a total of 19,750 MSM participants were surveyed from 14 surveillance points in Jiangsu, ranging from 3,061 to 4,766 per year. Among the surveyed individuals, approximately $80.0 \%$ were residents, and $20.0 \%$ were migrants. The proportion of residents increased from $75.6 \%$ in 2010 to $82.7 \%$ in 2012 and then decreased to $80.5 \%$ in 2014 .

The average age of the participant was $30.9 \pm 10.4$ years old. For participants under the age of 30 , the proportion of migrants (64.5\%) was higher than residents (53.6\%). Approximately $58.8 \%$ of residents and $69.7 \%$ of migrants reported never having been married. About three-quarters of all participants had at least attained senior high school, with 79.4\% among residents and 71.5\% among migrants, respectively. Among both residents and migrants, the most common location for meeting partners was the Internet followed by bars and clubs. Residents were somewhat more likely than migrants to meet partners on the Internet, while migrants were more likely than residents to meet partners at bath centers (Table 1 ).

Sexual Risk Behaviors. About four-fifths of the participants had engaged in anal sex in the last six months, with an increasing trend among both residents and migrants during the study period $(\mathrm{P}<0.01, \mathrm{AOR}=1.24$, 95\% CI:1.09-1.40 in residents; AOR $=1.47,95 \%$ CI:1.15-1.87 in-migrants). Increases were observed among resident MSM in rates of condom use during most recent anal sex with a male partner $(\mathrm{P}<0.01, \mathrm{AOR}=1.29,95 \%$ $\mathrm{CI} 1.13-1.46)$ and consistent condom use during anal sex in the last six months $(\mathrm{P}<0.01, \mathrm{AOR}=1.19,95 \%$ CI:1.05-1.34), while no statistically significant changes in these two behaviors were observed among migrants. However, the proportion reporting these three behaviors in migrants was higher than in residents for the majority of the surveyed period.

The trend in commercial anal sex for both residents and migrants decreased during the study period $(\mathrm{P}<0.01)$, with a somewhat higher proportion of migrants $(7.2 \%)$ participating, as compared to residents $(4.4 \%)$.

About 29.3\% (5,777/19,700) participants indicated that they had engaged in vaginal intercourse with a female at least once in the last six months. The proportion of participants reporting vaginal sex decreased among migrants $(\mathrm{P}<0.01, \mathrm{AOR}=0.61,95 \% \mathrm{CI}: 0.47-0.78)$. The proportions of residents who used a condom during their most recent vaginal intercourse increased from $42.0 \%$ in 2010 to $50.2 \%$ in $2014(\mathrm{P}<0.01, \mathrm{AOR}=1.48,95 \%$ CI: $1.22-1.80)$

A significant decreasing trend of drug use was observed in both residents (AOR $=0.57,95 \% \mathrm{CI}: 0.34-0.96$ ) and migrants $(\mathrm{AOR}=0.39,95 \% \mathrm{CI}: 0.17-0.88)$. While the proportion who received any HIV-related services increased consistently over time in both residents $(\mathrm{AOR}=1.45,95 \% \mathrm{CI}: 1.29-1.64)$ and migrants $(\mathrm{AOR}=1.48$, 95\% CI:1.18-1.85) (Table 2). 


\begin{tabular}{|c|c|c|c|c|c|c|c|c|c|c|c|c|}
\hline \multirow{2}{*}{ Variables } & \multirow{2}{*}{\begin{tabular}{|l|}
2010 \\
$(\%)$ \\
\end{tabular}} & \multirow{2}{*}{\begin{tabular}{|l|}
2011 \\
$(\%)$ \\
\end{tabular}} & \multirow{2}{*}{$\begin{array}{l}2012 \\
(\%)\end{array}$} & \multirow{2}{*}{\begin{tabular}{|l|}
2013 \\
$(\%)$ \\
\end{tabular}} & \multirow{2}{*}{$\begin{array}{l}2014 \\
(\%)\end{array}$} & \multirow{2}{*}{\begin{tabular}{|l|} 
Overall \\
$(\%)$
\end{tabular}} & \multirow{2}{*}{$\begin{array}{l}P \text { for } \\
\text { Trend }\end{array}$} & \multirow{2}{*}{\multicolumn{2}{|c|}{$\begin{array}{l}\text { Crude OR }{ }^{*} \\
(95 \% \text { CI })\end{array}$}} & \multirow{2}{*}{\multicolumn{2}{|c|}{$\begin{array}{l}\text { Adjusted OR* } \\
(95 \% \mathrm{CI})\end{array}$}} & \multirow{2}{*}{\begin{tabular}{|l|}
$P$ for \\
Adjusted Model
\end{tabular}} \\
\hline & & & & & & & & & & & & \\
\hline \multicolumn{13}{|c|}{ Engaged in anal sex in the last six months } \\
\hline Residents & 76.2 & 78.2 & 73.8 & 77.8 & 79.6 & 77.2 & $<0.01$ & 1.22 & $(1.08-1.38)$ & 1.24 & $(1.09-1.40)$ & $<0.01$ \\
\hline Migrants & 76.5 & 83.6 & 84.7 & 83.6 & 82.4 & 82.2 & $<0.01$ & 1.44 & $(1.13-1.83)$ & 1.47 & $(1.15-1.87)$ & $<0.01$ \\
\hline All & 76.3 & 79.4 & 75.7 & 78.9 & 80.2 & 78.2 & $<0.01$ & & & & & \\
\hline \multicolumn{13}{|c|}{ Used condom during last anal intercourse with male } \\
\hline Residents & 65.0 & 72.9 & 72.4 & 72.2 & 70.4 & 70.9 & $<0.01$ & 1.28 & $(1.13-1.45)$ & 1.29 & $(1.13-1.46)$ & $<0.01$ \\
\hline Migrants & 68.3 & 71.4 & 72.8 & 67.6 & 69.9 & 69.9 & 0.43 & 1.08 & $(0.85-1.36)$ & 1.08 & $(0.86-1.37)$ & 0.51 \\
\hline All & 65.8 & 72.6 & 72.5 & 71.2 & 70.3 & 70.7 & 0.02 & & & & & \\
\hline \multicolumn{13}{|c|}{ Consistent condom use during anal sex with male in the last six months } \\
\hline Residents & 36.7 & 44.0 & 41.9 & 43.0 & 40.9 & 41.5 & 0.06 & 1.19 & $(1.06-1.35)$ & 1.19 & $(1.05-1.34)$ & $<0.01$ \\
\hline Migrants & 43.3 & 45.9 & 47.3 & 40.1 & 41.7 & 43.5 & 0.06 & 0.93 & $(0.75-1.16)$ & 0.94 & $(0.75-1.17)$ & 0.55 \\
\hline All & 38.3 & 44.5 & 43.0 & 42.4 & 41.0 & 41.9 & 0.29 & & & & & \\
\hline \multicolumn{13}{|c|}{ Engaged in commercial anal sex with male in the last six months } \\
\hline Residents & 6.3 & 5.2 & 3.8 & 3.0 & 4.5 & 4.4 & $<0.01$ & 0.70 & $(0.54-0.90)$ & 0.75 & $(0.58-0.98)$ & 0.03 \\
\hline Migrants & 10.8 & 9.0 & 5.9 & 3.5 & 7.3 & 7.2 & $<0.01$ & 0.66 & $(0.45-0.96)$ & 0.69 & $(0.47-1.02)$ & 0.07 \\
\hline All & 7.4 & 6.1 & 4.2 & 3.1 & 5.0 & 5.0 & $<0.01$ & & & & & \\
\hline \multicolumn{13}{|c|}{ Used condom during last commercial anal intercourse with male } \\
\hline Residents & 75.2 & 83.0 & 68.7 & 79.2 & 85.8 & 78.9 & 0.05 & 1.99 & $(1.03-3.84)$ & 1.98 & $(1.01-3.88)$ & 0.05 \\
\hline Migrants & 86.7 & 90.4 & 67.6 & 79.2 & 82.1 & 82.5 & 0.12 & 0.71 & $(0.26-1.95)$ & 0.70 & $(0.24-2.00)$ & 0.50 \\
\hline All & 79.4 & \begin{tabular}{|l|}
85.4 \\
\end{tabular} & 68.4 & 79.2 & \begin{tabular}{|l|l|}
84.7 \\
\end{tabular} & 80.0 & 0.26 & & & & & \\
\hline Consisten & ondom & use dur & g com & nercial & nal sex & the last $\mathrm{s}$ & month & ith ma & & & & \\
\hline Residents & 47.7 & \begin{tabular}{|l|l|}
60.7 \\
\end{tabular} & 42.7 & \begin{tabular}{|l|}
62.8 \\
\end{tabular} & \begin{tabular}{|l|l|}
49.3 \\
\end{tabular} & 52.1 & 0.48 & 1.07 & $(0.64-1.77)$ & 1.08 & $(0.64-1.81)$ & 0.79 \\
\hline Migrants & 63.3 & 67.3 & 47.4 & 41.7 & 58.9 & 58.3 & 0.11 & 0.83 & $(0.39-1.76)$ & 0.67 & $(0.30-1.47)$ & 0.32 \\
\hline All & 53.3 & \begin{tabular}{|l|}
62.9 \\
\end{tabular} & 44.0 & 57.8 & 52.1 & 54.0 & 0.23 & & & & & \\
\hline Engaged ir & aginal & ex in th & last six & month & & & & & & & & \\
\hline Residents & 30.2 & 28.6 & 33.5 & 28.4 & 29.7 & 30.2 & 0.23 & 0.98 & $(0.87-1.09)$ & \begin{tabular}{|l|}
0.89 \\
\end{tabular} & $(0.79-1.02)$ & \begin{tabular}{|l|}
0.08 \\
\end{tabular} \\
\hline Migrants & 30.7 & 28.3 & 29.6 & 22.3 & 20.0 & 25.8 & $<0.01$ & 0.56 & $(0.45-0.71)$ & 0.61 & $(0.47-0.78)$ & $<0.01$ \\
\hline All & 30.3 & 28.6 & 32.8 & 27.2 & 27.8 & 29.3 & $<0.01$ & & & & & \\
\hline Used cond & a durin & last va & inal int & rcours & & & & & & & & \\
\hline Residents & 42.0 & 49.3 & 59.0 & 55.6 & 50.2 & 52.2 & $<0.01$ & 1.39 & $(1.15-1.69)$ & 1.48 & $(1.22-1.80)$ & $<0.01$ \\
\hline Migrants & 44.2 & \begin{tabular}{|l|}
46.9 \\
\end{tabular} & 61.0 & 58.1 & 48.6 & 51.7 & 0.03 & 1.19 & $(0.81-1.76)$ & 1.26 & $(0.84-1.89)$ & 0.27 \\
\hline All & 42.5 & 48.8 & 59.3 & 56.0 & 50.0 & 52.1 & $<0.01$ & & & & & \\
\hline Consisten & ondom & use dur & g vagir & al sex i & the las & ix months & & & & & & \\
\hline Residents & 24.5 & 28.3 & 21.8 & 25.6 & 24.7 & 24.7 & 0.36 & 1.01 & $(0.81-1.26)$ & 1.05 & $(0.84-1.32)$ & 0.68 \\
\hline Migrants & 27.6 & 30.6 & 29.1 & 36.9 & 30.3 & 30.7 & 0.13 & 1.14 & $(0.74-1.75)$ & 1.15 & $(0.73-1.80)$ & 0.55 \\
\hline All & 25.3 & 28.8 & 23.0 & 27.3 & 25.5 & 25.8 & 0.43 & & & & & \\
\hline Used drug & lifetim & & & & & & & & & & & \\
\hline Residents & 1.3 & 1.3 & 0.9 & 0.7 & 0.7 & 0.9 & $<0.01$ & 0.52 & $(0.31-0.88)$ & 0.57 & $(0.34-0.96)$ & 0.04 \\
\hline Migrants & 2.4 & 1.0 & 1.2 & 0.9 & 1.0 & 1.3 & $<0.01$ & 0.40 & $(0.18-0.89)$ & 0.39 & $(0.17-0.88)$ & 0.02 \\
\hline All & 1.6 & 1.3 & 0.9 & 0.7 & 0.8 & 1.0 & $<0.01$ & & & & & \\
\hline Received a & HIV-r & lated $s$ & vices ir & last ye & & & & & & & & \\
\hline Residents & 72.5 & 78.2 & 76.7 & \begin{tabular}{|l|}
81.7 \\
\end{tabular} & 79.4 & 78.1 & $<0.01$ & 1.47 & $(1.30-1.66)$ & 1.45 & $(1.29-1.64)$ & $<0.01$ \\
\hline Migrants & 71.1 & \begin{tabular}{|l|}
75.3 \\
\end{tabular} & 73.4 & \begin{tabular}{|l|}
82.1 \\
\end{tabular} & \begin{tabular}{|l|l|}
78.4 \\
\end{tabular} & 76.3 & $<0.01$ & 1.48 & $(1.19-1.85)$ & 1.48 & $(1.18-1.85)$ & $<0.01$ \\
\hline All & 72.1 & 77.6 & 76.1 & 81.8 & 79.2 & 77.7 & $<0.01$ & & & & & \\
\hline HIV preva & & & & & & & & & & & & \\
\hline Residents & 8.1 & 7.1 & 9.0 & 7.3 & \begin{tabular}{|l|}
8.8 \\
\end{tabular} & 8.1 & 0.13 & 1.10 & $(0.91-1.32)$ & 1.12 & $(0.93-1.36)$ & 0.23 \\
\hline Migrants & 11.1 & 10.9 & 12.1 & 14.1 & 9.2 & 11.4 & 0.34 & 0.81 & $(0.59-1.11)$ & 0.83 & $(0.60-1.14)$ & 0.25 \\
\hline All & 8.9 & \begin{tabular}{|l|}
7.9 \\
\end{tabular} & 9.6 & 8.6 & 8.9 & 8.8 & 0.30 & & & & & \\
\hline Syphilis in & tion ra & & & & & & & & & & & \\
\hline Residents & 12.8 & \begin{tabular}{|l|}
8.9 \\
\end{tabular} & 6.5 & 5.5 & 6.5 & 7.6 & $<0.01$ & 0.48 & $(0.40-0.57)$ & 0.48 & $(0.40-0.57)$ & $<0.01$ \\
\hline Migrants & 15.4 & 9.4 & 7.9 & 5.8 & 8.1 & 9.2 & $<0.01$ & 0.48 & $(0.35-0.66)$ & 0.48 & $(0.35-0.65)$ & $<0.01$ \\
\hline All & 13.4 & 9.0 & 6.8 & 5.5 & \begin{tabular}{|l|}
6.8 \\
\end{tabular} & \begin{tabular}{|l|}
7.9 \\
\end{tabular} & $<0.01$ & & & & & \\
\hline
\end{tabular}

Table 2. Behaviors, Receipt of Services and Serological Detection of MSM Changing across time during 2010 and 2014 in Jiangsu $(\mathrm{N}=19,750)$. Note: ${ }^{*}$ Odds Ratio was calculated by direct comparing the year 2014 to the year 2010 (reference group); *Multivariate models were adjusted for the following variables: age, marital status (ref $=$ married), education. $\mathrm{P}<0.01$. 
A

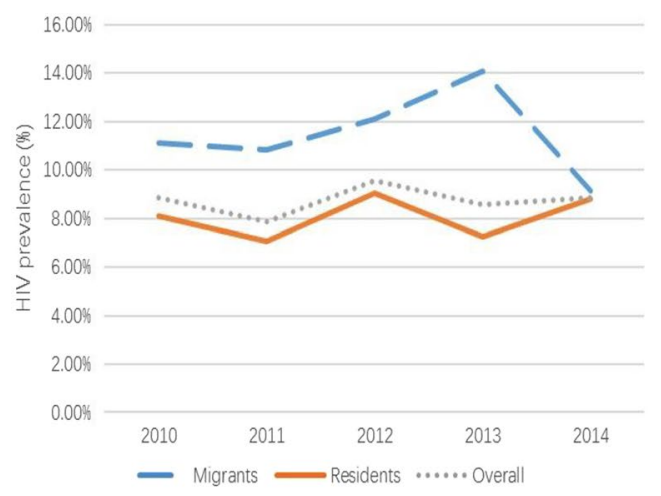

B

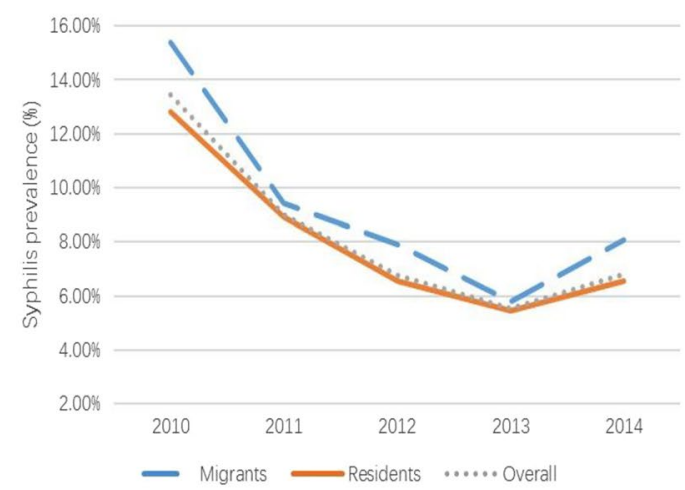

Figure 1. Trend of HIV (A) and syphilis (B) prevalence (overall, migrants, residents) for the participants during 2010 to 2014 in Jiangsu. (Created by Microsoft Excel, Redmond, Washington, the United States available at https://www.microsoftstore.com.cn/c/office?Icid=StoreNavi_Office).

HIV/Syphilis Prevalence and Trend. 1738 participants tested positive for HIV, with an overall HIV prevalence of $8.8 \%$. There was no obvious change in HIV prevalence among the two groups during the study period. However, it is important to note that the HIV infection rate was higher among migrants (11.4\%), as compared to residents (8.1\%).

While HIV prevalence was stable, the prevalence of syphilis decreased significantly (from $12.8 \%$ in 2010 to $6.5 \%$ in $2014, \mathrm{P}<0.01)$ among both resident $\mathrm{MSM}(\mathrm{P}<0.01, \mathrm{AOR}=0.48 ; 95 \% \mathrm{CI}: 0.40-0.57)$ and migrant MSM $(\mathrm{P}<0.01, \mathrm{AOR}=0.48 ; 95 \%$ CI: $0.35-0.65)$. (Table 2$)$ Syphilis prevalence among migrants was also consistently higher than residents during the study period (Fig. 1).

Behaviors correlated with HIV status. Compared to HIV-negative participants, the majority of MSM in the HIV-positive group had more anal sex in last six months (Table 3). From 2011 to 2014, a higher proportion of HIV-negative MSM used a condom during their last anal sex, and similar results were also observed for consistent condom use during the last six months. In addition, a significantly smaller proportion of HIV-positive participants engaged in commercial anal sex during the study period. Vaginal sex in the last six months was associated with HIV infections in the 2010 and 2012 surveys, while consistent condom use during vaginal sex in the last six months was associated with HIV infection in 2014. We also found that there was a link between lifetime drug use and HIV infection among HIV-positive MSM in 2011 and 2014 surveys (Table 3).

Geographic distributions. Geographic distribution of HIV, syphilis seropositivity in 13 sampling cities during 2014 was presented in Fig. 2, color-coded to represent the geographical differences in HIV and syphilis prevalence. Overall, Suzhou had the highest HIV prevalence $(>16 \%)$ in Jiangsu province, and seven cities had HIV infection rates over 10\%. For syphilis, Nanjing and Huai'an reported prevalence over 10\%. Compared to Southern Jiangsu, migrants in Central Jiangsu and residents in Northern Jiangsu has lower HIV prevalence, while people in Central Jiangsu had the lowest syphilis prevalence (Table 4).

\section{Discussion}

Our study found a higher prevalence of HIV and syphilis and higher rates of some high-risk behaviors in migrant MSM than in resident MSM. Knowledge of trends of HIV/STI prevalence and related sexual behaviors in migrant and resident MSM is critical for HIV/STI control efforts. In China, many HIV testing and care programs are targeted toward the general MSM population ${ }^{4,30,31}$, but few focus specifically on migrant MSM. This study extends the literature by exploring the trend of HIV and syphilis prevalence, related sexual behaviors, and geological distribution among MSM in Jiangsu. From the study, we found a decreasing trend of syphilis prevalence, while HIV prevalence was consistent.

In China, migrant population is often seen as poor, poorly educated, rural farmers or laborers who seek for a better job in large cities ${ }^{32}$. In our study, one-fifth of MSM participants is migrants. When they are living in a new city without sufficient HIV knowledge, family discipline and social support, they are more prone to make a sexual decision without constraint ${ }^{12,25,33}$, and more likely to engage in high-risk sexual behaviors and acquire HIV/ STIs ${ }^{34-36}$. The results indicated that only resident MSM had an increasing trend in condom use during last anal sex and inconsistent condom use in last six months, while migrant MSM did not show a consistent trend across time. Our study also found that compared with resident MSM, migrant MSM had higher HIV and syphilis prevalence, more frequent anal sexual behaviors, and higher lifetime drug use rates, whichever it is, consistent with a previous study of MSM in China ${ }^{37}$. Further studies on evaluating which factors had contributed to the high HIV and syphilis infections among migrants are needed.

The overall HIV prevalence from our study was about $8.8 \%$, which was higher than a previous survey in Jiangsu ${ }^{38}$ and higher than findings from two meta-analyses (6.5\% in $2013^{39}$ and $7.7 \%$ in $2013^{3}$, respectively). HIV prevalence among surveyed MSM in Jiangsu was higher than that of a cross-sectional survey ${ }^{32}$ and five years of accompanying analysis on HIV/AIDS in China ${ }^{2}$. Unlike findings from other studies ${ }^{4,18,40}$, the trend of HIV 


\begin{tabular}{|c|c|c|c|c|c|c|c|c|c|c|c|c|}
\hline \multirow{2}{*}{\begin{tabular}{|l|} 
Variable \\
Engaged i \\
\end{tabular}} & \multicolumn{2}{|c|}{$2010(\%)$} & \multicolumn{2}{|c|}{$2011(\%)$} & \multicolumn{2}{|c|}{$2012(\%)$} & \multicolumn{2}{|c|}{$2013(\%)$} & \multicolumn{2}{|c|}{$2014(\%)$} & \multicolumn{2}{|c|}{ Overall } \\
\hline & nal sex & $\mathrm{n}$ the last six $\mathrm{m}$ & ths & & & & & & & & & \\
\hline \multirow{2}{*}{$\begin{array}{l}\text { HIV- } \\
\text { HIV+ }\end{array}$} & 76.2 & $(2121 / 2783)$ & 78.8 & $(2408 / 3057)^{\mathrm{a}}$ & 75.0 & $(2960 / 3948)^{\mathrm{a}}$ & 78.2 & $(3007 / 3846)^{\mathrm{a}}$ & 80.1 & $(3485 / 4351)$ & 77.7 & $(13981 / 17985)^{a}$ \\
\hline & 76.4 & $(207 / 271)$ & 86.2 & $(225 / 261)$ & 82.3 & $(345 / 419)$ & 87.0 & $(314 / 361)$ & 81.1 & $(344 / 424)$ & 82.7 & $(1435 / 1736)$ \\
\hline \multicolumn{13}{|c|}{ Used condom during last anal intercourse with male } \\
\hline HIV- & 66.1 & $(1380 / 2088)$ & 73.2 & $(1739 / 2377)^{\mathrm{a}}$ & 73.1 & $(2123 / 2904)^{\mathrm{a}}$ & 72.4 & $(2173 / 3001)^{\mathrm{a}}$ & 71.3 & $(2475 / 3473)^{\mathrm{a}}$ & 71.4 & $(9890 / 13843)^{\mathrm{a}}$ \\
\hline $\mathrm{HIV}+$ & 63.4 & $(128 / 202)$ & 66.5 & $(149 / 224)$ & 67.3 & $(231 / 343)$ & 60.1 & $(188 / 313)$ & 61.0 & $(210 / 344)$ & 63.5 & $(906 / 1426)$ \\
\hline \multicolumn{13}{|c|}{ Consistently used condom during anal sex in the last six months } \\
\hline HIV- & 38.6 & $(801 / 2073)$ & 45.6 & $(1084 / 2376)^{a}$ & 43.8 & $(1267 / 2891)^{\mathrm{a}}$ & 44.1 & $(1323 / 3002)^{\mathrm{a}}$ & 42.2 & $(1463 / 3463)^{a}$ & 43.0 & $(5938 / 13805)^{\mathrm{a}}$ \\
\hline $\mathrm{HIV}+$ & 34.8 & $(69 / 198)$ & 32.0 & $(71 / 222)$ & 35.8 & $(123 / 344)$ & 26.6 & $(83 / 312)$ & 28.2 & $(97 / 344)$ & 31.2 & $(443 / 1420)$ \\
\hline \multicolumn{13}{|c|}{ Engaged in commercial anal sex with male in the last six months } \\
\hline HIV- & 7.2 & $(150 / 2116)$ & 6.3 & $(151 / 2398)$ & 4.1 & $(122 / 2965)$ & 3.1 & $(92 / 3007)$ & 4.7 & $(165 / 3474)^{\mathrm{a}}$ & 4.9 & $(683 / 13960)$ \\
\hline $\mathrm{HIV}+$ & 8.8 & $(18 / 204)$ & 3.6 & $(8 / 224)$ & 5.3 & $(18 / 342)$ & 3.2 & $(10 / 314)$ & 7.8 & $(27 / 344)$ & 5.7 & $(81 / 1428)$ \\
\hline \multicolumn{13}{|c|}{ Used condom during last commercial anal intercourse } \\
\hline HIV- & 79.7 & $(118 / 148)$ & 85.4 & $(129 / 151)$ & 67.8 & $(82 / 121)$ & 80.2 & $(73 / 91)$ & 89.0 & $(145 / 163)^{\mathrm{a}}$ & 81.2 & $(547 / 674)^{\mathrm{a}}$ \\
\hline $\mathrm{HIV}+$ & 76.5 & $(13 / 17)$ & 85.7 & $(6 / 7)$ & 73.3 & $(11 / 15)$ & 70.0 & $(7 / 10)$ & 59.3 & $(16 / 27)$ & 69.7 & $(53 / 76)$ \\
\hline \multicolumn{13}{|c|}{ Consistently used condom during commercial anal sex in the last six months } \\
\hline HIV- & 51.0 & $(76 / 146)^{\mathrm{a}}$ & 62.3 & $(94 / 151)$ & 43.1 & $(50 / 116)$ & 58.7 & $(54 / 92)$ & 55.8 & $(91 / 163)^{\mathrm{a}}$ & 54.4 & $(365 / 671)$ \\
\hline HIV+ & 72.2 & $(13 / 18)$ & 75.0 & $(6 / 8)$ & 50.0 & $(9 / 18)$ & 50.0 & $(5 / 10)$ & 29.6 & $(8 / 27)$ & 50.6 & $(41 / 81)$ \\
\hline \multicolumn{13}{|c|}{ Engaged in vaginal sex in the last six months } \\
\hline HIV- & 29.6 & $(823 / 2777)^{\mathrm{a}}$ & 28.4 & $(869 / 3056)$ & 33.5 & $(1324 / 3951)^{\mathrm{a}}$ & 27.5 & $(1057 / 3843)$ & 28.2 & $(1226 / 4343)$ & 29.5 & $(5299 / 17970)$ \\
\hline $\mathrm{HIV}+$ & 37.4 & $(101 / 270)$ & 30.3 & $(79 / 261)$ & 26.1 & $(109 / 417)$ & 24.2 & $(87 / 360)$ & 24.2 & $(102 / 422)$ & 27.6 & $(478 / 1730)$ \\
\hline \multicolumn{13}{|c|}{ Used condom during last vaginal intercourse } \\
\hline HIV- & 42.5 & $(348 / 819)$ & 48.9 & $(417 / 853)$ & 59.9 & $(777 / 1297)$ & 56.2 & $(591 / 1052)$ & 49.6 & $(606 / 1221)$ & 52.3 & $(2739 / 5242)$ \\
\hline $\mathrm{HIV}+$ & 42.4 & $(42 / 99)$ & 48.1 & $(38 / 79)$ & 53.2 & $(58 / 109)$ & 54.0 & $(47 / 87)$ & 55.9 & $(57 / 102)$ & 50.8 & $(242 / 476)$ \\
\hline \multicolumn{13}{|c|}{ Consistently used condom during vaginal sex in the last six months } \\
\hline HIV- & 24.8 & $(200 / 808)$ & 29.1 & $(247 / 849)$ & 22.8 & $(294 / 1292)$ & 26.7 & $(281 / 1054)$ & 24.7 & $(301 / 1218)^{\mathrm{a}}$ & 25.3 & $(1323 / 5221)^{\mathrm{a}}$ \\
\hline HIV + & 28 & $(28 / 94)$ & 25.3 & $(20 / 79)$ & 26.2 & $(28 / 107)$ & 35.6 & $(31 / 87)$ & 34.7 & $(35 / 101)$ & 30.3 & $(142 / 468)$ \\
\hline \multicolumn{13}{|c|}{ Used drug in lifetime } \\
\hline HIV- & 1.7 & $(46 / 2782)$ & 1.2 & $(35 / 3040)^{\mathrm{a}}$ & 0.9 & $(37 / 3949)$ & 0.7 & $(26 / 3845)$ & 0.6 & $(26 / 4334)^{\mathrm{a}}$ & 0.9 & $(170 / 17950)^{\mathrm{a}}$ \\
\hline $\mathrm{HIV}+$ & 1.1 & $(3 / 270)$ & 2.7 & $(7 / 261)$ & 0.7 & $(3 / 415)$ & 1.1 & $(4 / 361)$ & 2.4 & $(10 / 421)$ & 1.6 & $(27 / 1728)$ \\
\hline \multicolumn{13}{|c|}{ Received any HIV-related services in last year } \\
\hline HIV- & 71.5 & $(1997 / 2792)$ & 77.7 & $(2391 / 3076)$ & 76.4 & $(3028 / 3965)$ & 82.3 & $(3166 / 3846)^{\mathrm{a}}$ & 80.1 & $(3485 / 4351)^{\mathrm{a}}$ & 78.0 & $(14067 / 18030)^{\mathrm{a}}$ \\
\hline $\mathrm{HIV}+$ & 78.7 & $(214 / 272)$ & 76.0 & $(199 / 262)$ & 73.7 & $(309 / 419)$ & 76.5 & $(276 / 361)$ & 71.0 & $(301 / 424)$ & \begin{tabular}{|l|}
74.7 \\
\end{tabular} & \begin{tabular}{|l}
$(1299 / 1738)$ \\
\end{tabular} \\
\hline
\end{tabular}

Table 3. HIV-Related Services and Behaviors for HIV negative and positive MSM* in Jiangsu (2010-2014,

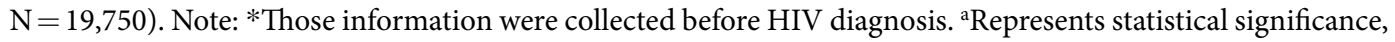
$\mathrm{P}<0.05$.

prevalence in our study did not change during the study period but remained at a high level. This high prevalence is particularly concerning given our study spanned five years and included a large sample of MSM.

Syphilis prevalence among MSM in our study was about $7.9 \%$, which was much lower than several previous studies of Jiangsu ${ }^{28,38,41}$ and also lower than other previous surveys of China ${ }^{32,42}$. Unlike the trend of HIV prevalence, syphilis prevalence decreased consistently, which is consistent with the previous literature ${ }^{18}$. One potential explanation for this phenomenon is the expansion of syphilis testing and control programs as well as the improving of other healthcare service ${ }^{28,40}$, while syphilis is a curable disease but not HIV. Syphilis prevention strategies included early screening and appropriate treatments ${ }^{6}$. Among the high-risk population, the coverage of screening and treatment for syphilis were increased in recent years in China ${ }^{4}$. Several MSM-targeted interventions were implemented in parallels, such as $100 \%$ condom promotion, behavioral intervention, and expansion of syphilis testing and treatment ${ }^{7}$. These measures may have led to a decline in syphilis prevalence among MSM. These prevention strategies may also have contributed to HIV control, which may explain why HIV epidemic was stable during the study period. As we know, Syphilis infection is at greater risk of HIV infection ${ }^{43}$, the high rate of Syphilis may suggest an increasing rate of HIV among MSM. However, the geographic distribution of HIV and Syphilis are not consistent in Jiangsu province, while the specific reasons for this inconsistent are still not clear, curable of syphilis could be one potential reason ${ }^{44}$.

Our study found that HIV-positive individuals are more likely to engage in anal sex, but less likely to use condoms consistently, as compared to HIV-negative participants. Previous studies demonstrated that condomless sexual activity is a risk factor for HIV infections in $\mathrm{MSM}^{45,46}$, particularly these participants who knows they were HIV-positive when a condom was not used during sexual intercourse, the virus will be transmitted easily through the anal intercourse ${ }^{47}$. Our study also indicated that even migrant MSM and resident MSM have comparable behaviors on condom use, migrant MSM tend to have a high prevalence of HIV. One potential reason for this phenomenon 
A

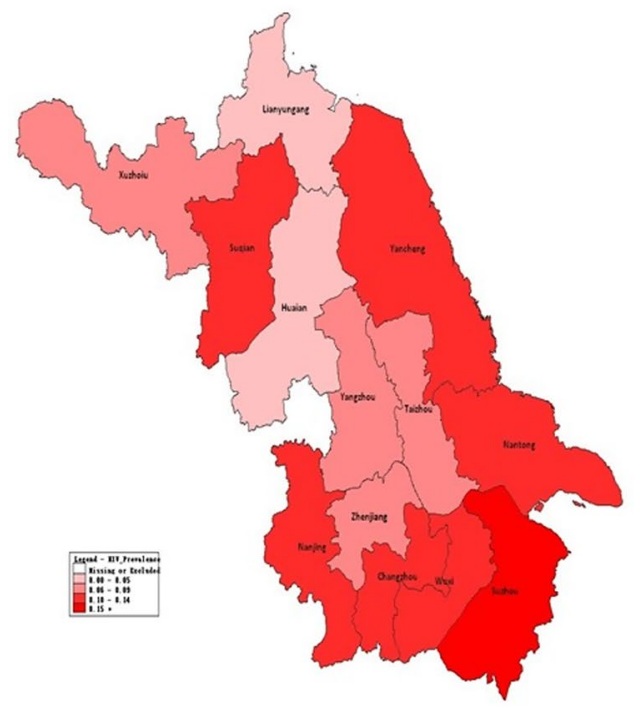

B

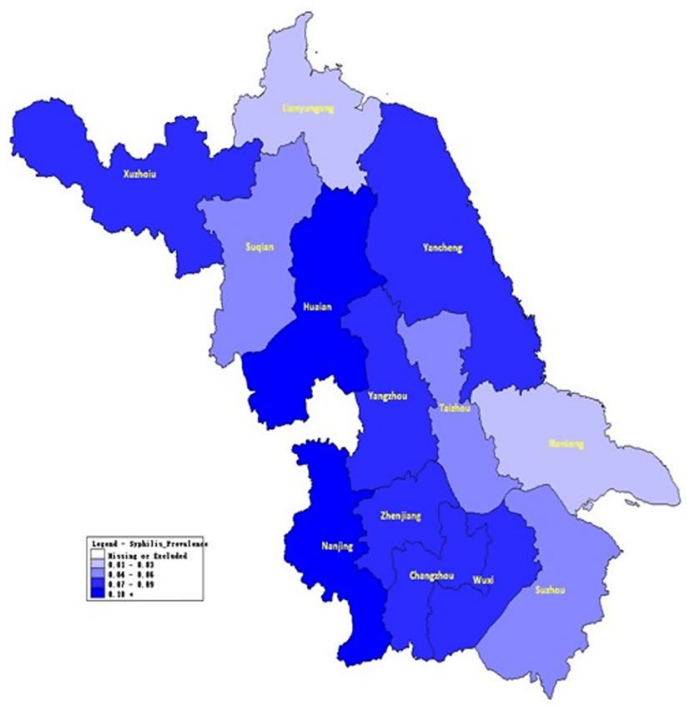

Figure 2. Map of Jiangsu showing the geographical distribution of HIV (A) and syphilis (B) prevalence among MSM in 2014 in 13 cities. (Created by Epi Info 3.5.1 software, Centers for Disease Control and Prevention, Atlanta, GA, USA, available at http://www.cdc.gov/epiinfo/index.html).

\begin{tabular}{|c|c|c|c|c|c|c|c|}
\hline \multirow[b]{2}{*}{ Variable } & \multirow[b]{2}{*}{ Region $^{a}$} & \multicolumn{2}{|l|}{ Migrants } & \multicolumn{2}{|l|}{ Residents } & \multicolumn{2}{|l|}{ Overall } \\
\hline & & $\begin{array}{l}\text { Crude OR } \\
(95 \% \text { CI })\end{array}$ & \begin{tabular}{|l} 
Adjusted $\mathrm{OR}^{\#}$ \\
$(95 \% \mathrm{CI})$
\end{tabular} & $\begin{array}{l}\text { Crude OR } \\
(95 \% \text { CI })\end{array}$ & $\begin{array}{l}\text { Adjusted } \mathrm{OR}^{\#} \\
(95 \% \mathrm{CI})\end{array}$ & $\begin{array}{l}\text { Crude OR } \\
(95 \% \text { CI })\end{array}$ & \begin{tabular}{|l} 
Adjusted OR \\
$(95 \%$ CI $)$
\end{tabular} \\
\hline \multirow{3}{*}{ HIV } & South Jiangsu & \multicolumn{6}{|l|}{ Ref } \\
\hline & Central Jiangsu & $0.37(0.18-0.75)^{*}$ & $0.3(0.14-0.64)^{*}$ & $0.88(0.67-1.17)$ & $0.87(0.65-1.16)$ & $0.77(0.59-0.99)$ & $0.74(0.57-0.96)$ \\
\hline & North Jiangsu & $0.51(0.20-1.29)$ & $0.52(0.20-1.33)$ & $0.63(0.49-0.82)^{*}$ & $0.57(0.43-0.74)^{*}$ & $0.61(0.48-0.78)^{*}$ & $0.58(0.45-0.74)^{*}$ \\
\hline Syphilis & North Jiangsu & $0.8(0.33-1.92)$ & $0.82(0.34-1.97)$ & $0.77(0.58-1.02)$ & $0.7(0.52-0.94)$ & $0.74(0.57-0.96)$ & $0.68(0.52-0.88)^{*}$ \\
\hline
\end{tabular}

Table 4. Odds ratios Between Human Immunodeficiency Virus (HIV) and Syphilis Among MSM in different

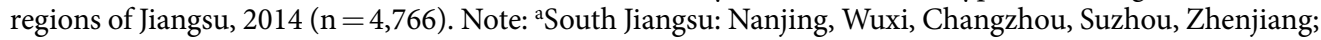
Central Jiangsu: Nantong, Yangzhou, Taizhou; North Jiangsu: Xuzhou, Lianyungang, Huaian, Yancheng, Suqian. "Odds Ratio was calculated by direct comparing region Central Jiangsu and North Jiangsu to South Jiangsu (reference group); Multivariate models were adjusted for the following variables: age, marital status (ref= married), education. *Represents statistical significance. $\mathrm{P}<0.01$.

is migrants tend to have more sexual partners and tend to have a set of sexual partners with greater STI risk behaviors ${ }^{26}$, such as more secondary sexual partners (partners other than the index) and more receptive anal intercourse.

Due to the unregistered residential status, migrant MSM tends to have less opportunity to access many healthcare services and are not well covered by many HIV/STI programs in most places in China ${ }^{48,49}$. For example, previous studies show that migrant MSM faces barriers to accessing adequate health services ${ }^{9,50,51}$. In addition, the healthcare system in China has lower rates of health services coverage for migrants as compared to residents, especially for HIV prevention services ${ }^{52}$. Meanwhile, the migrants are also rarely utilizing these medical resource $^{52}$. Therefore, more efforts are needed to promote interventions targeting migrant MSM. First, the public sector should increase the social supports and help to build and strengthen healthcare system to cover the whole population of China. Second, specific attention is required tailored behavioral interventions program on migrant MSM, like condom promotion, peer education. Third, a free and easily accessible HIV screening is necessary to be provided for migrant MSM as well as the treatment for HIV/STIs.

Our study has several limitations. First, because of the study design, lack of temporality prevented us from studying the relationship of demographic and high-risk factors for HIV/STI epidemics between residents and migrants. Second, MSM living with HIV with knowing status may be no longer willing to participate in sentinel surveillance, and the HIV prevalence reported in our study tends to be understated. Third, since most of our data were self-reported, social desirability bias can occur. Fourth, when participants recalled behavior over the last six months, misclassification of exposures may also occur. Last but not least, we did not consistently collect data on whether participants knew their HIV and syphilis status, which limited our ability to adjust this in our data analysis. 


\section{Conclusion}

This study suggests that prevalence of HIV among MSM in Jiangsu remains high, and HIV control remains an ongoing challenge in the future. In addition, this study demonstrates that migrant MSM compared to resident MSM were more likely to have high sexual risk behaviors. To decrease the HIV/STIs burden among MSM in Jiangsu, especially among migrant MSM, targeted intervention programs should be developed, and comprehensive intervention strategies should be enhanced to ensure that the needs of this high-risk population are met.

\section{References}

1. Beyrer, C. et al. Global epidemiology of HIV infection in men who have sex with men. The Lancet 380, 367-377 (2012).

2. Hei, F. et al. Epidemic characteristics of HIV/AIDS among men who have sex with men from 2006 to 2010 in China. Chin J Epidemical 33, 67-70 (2012).

3. Huang, Q., Li, Q., Li, Y. \& Zeng, G. Prevalence of HIV infection and syphilis, sexual behaviors and awareness of HIV/AIDS related knowledge among men who have sex with men in China: a Meta-analysis of data collected from 2010 to 2013. Chinese Journal of Epidemiology 36, 1297-1304 (2015).

4. Wang, B., Li, X., Stanton, B., Liu, Y. \& Jiang, S. Socio-demographic and behavioral correlates for HIV and syphilis infections among migrant men who have sex with men in Beijing, China. AIDS Care 25, 249-257 (2013).

5. Pan, X. et al. High prevalence of HIV among men who have sex with men in Zhejiang, China: a respondent-driven sampling survey. BMJ open 5, e008466 (2015).

6. Lau, J. T., Lin, C., Hao, C., Wu, X. \& Gu, J. Public health challenges of the emerging HIV epidemic among men who have sex with men in China. Public health 125, 260-265 (2011)

7. Feng, Y. et al. HIV/STD prevalence among men who have sex with men in Chengdu, China and associated risk factors for HIV infection. Journal of acquired immune deficiency syndromes 53(Suppl 1), S74-80 (2010).

8. Wang, B. et al. HIV-related risk behaviors and history of sexually transmitted diseases among male migrants who patronize commercial sex in China. Sexually transmitted diseases 34, 1-8 (2007).

9. Hong, Y. et al. Rural-to-urban migrants and the HIV epidemic in China. AIDS and behavior 10, 421-430 (2006).

10. Wong, D. F. \& Song, H. X. The resilience of migrant workers in Shanghai China: the roles of migration stress and meaning of migration. The International journal of social psychiatry 54, 131-143 (2008).

11. Li, X. et al. HIV/STD risk behaviors and perceptions among rural-to-urban migrants in China. AIDS Educ Prev 16, 538-556 (2004).

12. Wang, W. et al. Prevalence and risks for sexually transmitted infections among a national sample of migrants versus non-migrants in China. International journal of STD \& AIDS 21, 410-415 (2010).

13. Mi, G. et al. Hidden and Mobile: A Web-based Study of Migration Patterns of Men Who Have Sex With Men in China. Clinical infectious diseases: an official publication of the Infectious Diseases Society of America 62, 1443-1447 (2016).

14. Yang, X., Derlega, V. J. \& Luo, H. Migration, behaviour change and HIV/STD risks in China. AIDS Care 19, 282-288 (2007).

15. Li, X., Fang, X. \& Lin, D. HIV/STD risk behaviors and perceptions among rural-urban migrants in china. AIDS education and prevention: official publication of the International Society for AIDS Education 16, 538-556 (2004).

16. Ruan, S. et al. HIV prevalence and correlates of unprotected anal intercourse among men who have sex with men, Jinan, China. AIDS and behavior 12, 469-475 (2008).

17. Shi, T. X. et al. Study on the high risk behaviors related to AIDS among men who having sex with men in the floating population. Zhonghua liu xing bing xue za zhi=Zhonghua liuxingbingxue zazhi 30, 668-671 (2009).

18. Qin, Q. et al. Changing trend of HIV, Syphilis and Hepatitis C among Men Who Have Sex with Men in China. Scientific reports 6, 31081 (2016).

19. Tucker, J. D., Yin, Y. P., Wang, B., Chen, X. S. \& Cohen, M. S. An expanding syphilis epidemic in China: epidemiology, behavioural risk and control strategies with a focus on low-tier female sex workers and men who have sex with men. Sexually transmitted infections 87(Suppl 2), ii16-18 (2011).

20. Hu, H. et al. Increasing HIV Incidence among Men Who Have Sex with Men in Jiangsu Province, China: Results from Five Consecutive Surveys, 2011-2015. International journal of environmental research and public health 13 (2016).

21. Sentinel, C. H. Guidelines for Conducting HIV Sentinel Serosurveys among Pregnant Women and Other Groups.

22. Welfare, M.o.H.a.F. operational guidelines for HIV sentinel surveillance. (ed. Organisation, N.A.C.) 148 (India, 2008 ).

23. Services, U.S.D.o.H.a.H., (HHS-CDC), C.f.D.C.a.P., Team, G.A.P.G.S. \& WHO. Introduction to HIV, AIDS and STI Surveillance for Africa Region. (UCSF, USA, 2009).

24. Berg, C. J. et al. Prevalence and correlates of tobacco use among a sample of MSM in Shanghai, China. Nicotine \& tobacco research: official journal of the Society for Research on Nicotine and Tobacco 13, 22-28 (2011).

25. Bianchi, F. T. et al. The sexual experiences of Latino men who have sex with men who migrated to a gay epicentre in the USA. Culture, health \& sexuality 9, 505-518 (2007).

26. Wu, J., Wu, H., Li, P. \& Lu, C. HIV/STIs risks between migrant MSM and local MSM: a cross-sectional comparison study in China. PeerJ 4, e2169 (2016).

27. Zhou, Y. et al. Rates of HIV, syphilis, and HCV infections among different demographic groups of female sex workers in Guangxi China: Evidence from 2010 national sentinel surveillance data. AIDS Care 25, 1433-1441 (2013).

28. Fu, G. F. et al. The epidemic of HIV, syphilis, chlamydia and gonorrhea and the correlates of sexual transmitted infections among men who have sex with men in Jiangsu, China, 2009. PloS one 10, e0118863 (2015).

29. Cary. Institute The SAS system for Windows, (USA, 1996).

30. Tao, J. et al. Home-based HIV testing for men who have sex with men in China: a novel community-based partnership to complement government programs. PloS one 9, e102812 (2014)

31. Tucker, J. D. et al. Organizational characteristics of HIV/syphilis testing services for men who have sex with men in South China: a social entrepreneurship analysis and implications for creating sustainable service models. BMC infectious diseases 14, 601 (2014).

32. Wu, Z. et al. HIV and syphilis prevalence among men who have sex with men: a cross-sectional survey of 61 cities in China. Clinical infectious diseases: an official publication of the Infectious Diseases Society of America 57, 298-309 (2013).

33. Egan, J. E. et al. Migration, neighborhoods, and networks: approaches to understanding how urban environmental conditions affect syndemic adverse health outcomes among gay, bisexual and other men who have sex with men. AIDS and behavior 15(Suppl 1), S35-50 (2011).

34. Guo, Y., Li, X., Song, Y. \& Liu, Y. Bisexual behavior among Chinese young migrant men who have sex with men: implications for HIV prevention and intervention. AIDS Care 24, 451-458 (2012)

35. Hu, Z., Liu, H., Li, X., Stanton, B. \& Chen, X. HIV-related sexual behaviour among migrants and non-migrants in a rural area of China: role of rural-to-urban migration. Public health 120, 339-345 (2006).

36. Chen, X. S., Peeling, R. W., Yin, Y. P. \& Mabey, D. C. The epidemic of sexually transmitted infections in China: implications for control and future perspectives. BMC medicine 9, 111 (2011).

37. Zou, X. et al. Rural-to-urban migrants are at high risk of sexually transmitted and viral hepatitis infections in China: a systematic review and meta-analysis. BMC infectious diseases 14, 490 (2014).

38. Tucker, J. D., Chen, X. S. \& Peeling, R. W. Syphilis and social upheaval in China. The New England journal of medicine 362, 1658-1661 (2010). 
39. Zhou, Y. et al. Prevalence of HIV and syphilis infection among men who have sex with men in China: a meta-analysis. BioMed research international 2014, 620431 (2014).

40. Ruan, S. et al. Rising HIV prevalence among married and unmarried among men who have sex with men: Jinan, China. AIDS and behavior 13, 671-676 (2009).

41. Jiang, J. et al. High prevalence of sexually transmitted diseases among men who have sex with men in Jiangsu Province, China. Sexually transmitted diseases 33, 118-123 (2006).

42. Gao, L., Zhang, L. \& Jin, Q. Meta-analysis: prevalence of HIV infection and syphilis among MSM in China. Sexually transmitted infections 85, 354-358 (2009).

43. Rottingen, J. A., Cameron, D. W. \& Garnett, G. P. A systematic review of the epidemiologic interactions between classic sexually transmitted diseases and HIV: how much really is known? Sexually transmitted diseases 28, 579-597 (2001).

44. Zhang, D. et al. Enhancing HIV Testing and Treatment among Men Who Have Sex with Men in China: A Pilot Model with TwoRapid Tests, Single Blood Draw Session, and Intensified Case Management in Six Cities in 2013. PloS one 11, e0166812 (2016).

45. Ma, X., Zhang, Q., Zhao, J., He, X. \& Du, H. Feasibility of using respondent driven sampling in HIV/STD epidemiological survey among MSM. Chinese Journal of Aids \& Std 13, 311-313 (2007).

46. Liu, X. Y., Yan, H., Tang, W. \& ZHANG, M. Analysis on the risk factors of AIDS in the MSM in Nanjing, 2008. Jiangsu Journal Of Preventive Medicine 21, 7-11 (2010)

47. Tang, W. et al. Factors associated with unprotected anal intercourse among men who have sex with men: results from a respondent driven sampling survey in Nanjing, China, 2008. AIDS and behavior 17, 1415-1422 (2013).

48. Mao, H. et al. High incidence of HIV and syphilis among migrant men who have sex with men in Beijing, China: a prospective cohort study. BMJ open 4, e005351 (2014).

49. Chow, E. P. et al. HIV prevalence trends, risky behaviours, and governmental and community responses to the epidemic among men who have sex with men in China. BioMed research international 2014, 607261 (2014).

50. Todrys, K. W. \& Amon, J. J. Within but without: human rights and access to HIV prevention and treatment for internal migrants. Globalization and health 5, 17 (2009).

51. Zhang, L., Chow, E. P., Jahn, H. J., Kraemer, A. \& Wilson, D. P. High HIV prevalence and risk of infection among rural-to-urban migrants in various migration stages in China: a systematic review and meta-analysis. Sexually transmitted diseases 40, 136-147 (2013).

52. Wang, W., Muessig, K. E., Li, M. \& Zhang, Y. X. Networking activities and perceptions of HIV risk among male migrant market vendors in China. AIDS and behavior 18(Suppl 2), S142-151 (2014).

\section{Acknowledgements}

The authors acknowledge the staff members of HIV/AIDS sentinel surveillance system of Jiangsu, China, and all participants. The author also thanks Tiange P. Zhang for his help on English Editing. This study was supported by National Institutes of Health (NIAID 1R01AI114310, FIC 1D43TW009532), NIH Fogarty International Center (5R25TW009340), and UNC Center for AIDS Research (National Institute of Allergy and Infectious Diseases 5P30AI050410), and the Bill \& Melinda Gates Foundation to the MeSH Consortium (BMGF-OPP1120138).

\section{Author Contributions}

Y.C. and W.T. wrote the main manuscript text, W.T., L.C. and G.F. revised the manuscript, Y.C. and L.S. analyzed the data, X.L., J.X., H.H. and H.Y. collected the data. All authors reviewed the manuscript.

\section{Additional Information}

Competing Interests: The authors declare that they have no competing interests.

Publisher's note: Springer Nature remains neutral with regard to jurisdictional claims in published maps and institutional affiliations.

(c) Open Access This article is licensed under a Creative Commons Attribution 4.0 International

License, which permits use, sharing, adaptation, distribution and reproduction in any medium or format, as long as you give appropriate credit to the original author(s) and the source, provide a link to the Creative Commons license, and indicate if changes were made. The images or other third party material in this article are included in the article's Creative Commons license, unless indicated otherwise in a credit line to the material. If material is not included in the article's Creative Commons license and your intended use is not permitted by statutory regulation or exceeds the permitted use, you will need to obtain permission directly from the copyright holder. To view a copy of this license, visit http://creativecommons.org/licenses/by/4.0/.

(C) The Author(s) 2017 